\title{
Elaboração e validação de uma cartilha eletrônica para diagnóstico de desordens orais potencialmente malignas
}

\author{
Preparation and validation and of an electronic booklet for diagnosing pontentially malignant oral
} disorders

Elaboración y validación de un folleto electrónico para el diagnóstico transtornos oral es potencialmente malignos

\author{
Karla Pinheiro de Alencar Castro \\ ORCID: https://orcid.org/0000-0002-9416-9012 \\ Universidade Regional do Cariri, Brasil \\ E-mail: karlapalencar@hotmail.com \\ Sabrina Alaide Amorim Alves \\ ORCID: https://orcid.org/0000-0001-5831-4668 \\ Universidade Regional do Cariri, Brasil \\ E-mail: sabrina1995amorim@gmail.com \\ Evanira Rodrigues Maia \\ ORCID: https://orcid.org/0000-0001-9377-7430 \\ Universidade Regional do Cariri, Brasil \\ E-mail: evanira.maia@urca.br \\ Anna Karina Barros de Moraes Ramalho \\ ORCID: https://orcid.org/0000-0003-0909-1634 \\ Universidade Federal da Paraíba, João Pessoa, PB, Brasil \\ E-mail: annakabm@hotmail.com \\ Maria do Socorro Vieira Lopes \\ ORCID: https://orcid.org/0000-0003-1335-5487 \\ Universidade Regional do Cariri, Brasil \\ E-mail: socorro.lopes@urca.br
}

\begin{abstract}
Resumo
Objetivo: descrever o processo de elaboração e validação uma cartilha eletrônica para auxiliar no diagnóstico de desordens potencialmente malignas da cavidade oral. Método: estudo metodológico, desenvolvido mediante levantamento de dados por meio de uma revisão integrativa e com o público-alvo com aplicação de um questionário; construção da cartilha; qualificação do material através de validação por juízes especialistas da área de saúde e de comunicação/designer. Resultados: A primeira versão da cartilha conteve 23 páginas e na sua avaliação, o Índice de Validade de Conteúdo global foi de 0,97 entre os juízes especialistas; a aparência foi qualificada como "superior" com percentual de 98,4\%. Conclusão: a cartilha foi validada, podendo ser considerada um material válido e confiável com uma linguagem clara, layout e designer atraente, a qual pode ser utilizada por diferentes profissionais para auxiliar no diagnóstico precoce de desordens orais potencialmente malignas.
\end{abstract}

Palavras-chave: Neoplasias bucais; Tecnologia educacional; Diagnóstico precoce.

\begin{abstract}
Objective: to describe the process of elaboration and validation of an electronic booklet to assist in the diagnosis of potentially malignant disorders of the oral cavity. Method: methodological study, developed through data collection through an integrative review and with the target audience with the application of a questionnaire; construction of the booklet; qualification of the material through validation by expert judges in the field of health and communication/designer. Results: The first version of the booklet contained 23 pages and in its evaluation, the global Content Validation Index was 0.97 among expert judges; the appearance was qualified as 'superior' with a percentage of $98.4 \%$. Conclusion: the booklet was validated and can be considered a valid and reliable material with a clear language, layout and attractive design, which can be used by different professionals to assist in the early diagnosis of potentially malignant oral disorders.
\end{abstract}

Keywords: Oral neoplasms; Educational technology; Early diagnosis. 


\begin{abstract}
Resumen
Objetivo: describir el proceso de elaboración y validación de un folleto electrónico para ayudar en el diagnóstico de trastornos potencialmente malignos de la cavidad bucal. Método: estudio metodológico, desarrollado a través de la recolección de datos a través de una revisión integradora y con el público objetivo con la aplicación de un cuestionario; construcción del folleto; Calificación del material mediante validación por jueces expertos en el campo de la salud y la comunicación / diseñador. Resultados: La primera versión del folleto contenía 23 páginas y en su evaluación, el Índice de Validación de Contenido global fue de 0.97 entre jueces expertos; la apariencia fue calificada como 'superior' con un porcentaje del 98,4\%. Conclusión: el folleto fue validado y puede considerarse un material válido y confiable con un lenguaje claro, maquetación y diseño atractivo, que puede ser utilizado por diferentes profesionales para ayudar en el diagnóstico precoz de trastornos orales potencialmente malignos.
\end{abstract}

Palabras clave: Neoplasias buçales; Tecnologia Educacional; Diagnostico temprano.

\title{
1. Introdução
}

O câncer oral é uma neoplasia maligna entre as três primeiras de maior incidência e ainda o sexto tipo de tumor mais frequente mundialmente, sendo considerado um sério problema de saúde pública, representando cerca de $12 \%$ de todas as causas de morte no mundo (Inca, 2014).

No Brasil, para cada ano do triênio 2020-2022, estima-se 11.180 casos de câncer de boca em homens e 4.010 em mulheres. Quanto à mortalidade, atestou-se que 6.605 casos, sendo 5,120 em homens e 1.485 em mulheres (Inca, 2020).

Considerada como uma neoplasia extremamente agressiva, o câncer bucal exige reconhecimento das suas possíveis lesões precursoras, as "desordens potencialmente malignas", entre as mais importantes, a leucoplasia, a eritroplasia, a queilite actínica e o líquen plano oral, no intento de prevenir a sua progressão (OMS, 2005).

A importância do diagnóstico precoce, tanto das desordens potencialmente malignas quanto do Carcinoma de Células Escamosas (CCE), é definida por meio da taxa de mortalidade observada em estágios avançados da doença, o que torna o câncer bucal relevante em todo o mundo. Considera-se que entre os profissionais da área da saúde o Cirurgião-Dentista (CD) deve ser o mais apropriado para o diagnóstico precoce de câncer oral e das desordens orais potencialmente malignas. Atribuindo a esses profissionais a competência e capacidade de ter conhecimentos sobre os fatores de risco e as estratégias de detecção precoce do câncer de boca (Silva, 2018).

Ações de promoção e prevenção primária em saúde, rastreamento, monitoramento, diagnóstico precoce e tratamento do câncer bucal têm importância reconhecida, em que sua prevenção tem sido realizada, basicamente, por meio da orientação para eliminação ou redução de fatores de risco, como consumo de tabaco e álcool, e evitar exposição ao sol para os cânceres de lábio inferior, destacando-se como um método eficaz de combate à doença (Silveira, 2014; Brasil, 2018).

No entanto, evidencia-se um déficit na qualificação de cirurgiões - dentistas na Atenção Básica quanto ao conhecimento sobre os tipos mais comuns de câncer bucal e identificação das desordens ditas como potencialmente malignas o que geram impacto negativo, refletindo no cuidado e atenção da saúde da população e ainda, paralelamente, destaca-se uma tímida oferta de treinamentos sobre o tema, o que demonstra a necessidade de potencialização da abordagem mais abrangente do assunto (Falcão, 2010).

Dentre as estratégias utilizadas para Educação Permanente, encontra-se a oferta de materiais instrutivos para profissionais, em que se destacam manuais, guias e cartilhas instrutivas, funcionando como possíveis fontes de informações e consequentemente qualificação para os profissionais na detecção precoce e diagnósticos de agravos, frequentemente recomendadas no setor saúde e outros, em que as formas eletrônicas têm ganhado o espaço de modo progressivo e acelerado, em substituição, ou como uma opção a mais, em relação aos impressos (Carvalho, 2013; Ramos, 2018).

Ante ao exposto, destaca a importância da utilização de tecnologias educativas, que poderá constituir como uma ferramenta importante para o diagnóstico destas desordens, na promoção de saúde e reabilitação oral, o que é de suma importância para melhores chances no tratamento e maior sobrevida e qualidade de vida do paciente. Assim, o estudo teve 
como objetivo descrever o processo de elaboração e validação uma cartilha eletrônica para auxiliar no diagnóstico de desordens potencialmente malignas da cavidade oral.

\section{Metodologia}

Trata-se de um estudo metodológico desenvolvido mediante as etapas sugeridas por Echer (2005): 1) submissão do projeto qualificado ao Comitê de Ética e Pesquisa; 2) levantamento de dados na literatura e diagnóstico situacional com o público-alvo; 3) construção da cartilha, ilustrações, layout, design e textos; 4) validação com especialistas da área da saúde e especialistas técnicos da área de comunicação/ designer.

Inicialmente, realizou-se uma revisão integrativa, norteada pela seguinte questão norteadora: O que o cirurgiãodentista precisa saber sobre prevenção e diagnóstico precoce de desordens orais potencialmente malignas? Foram selecionados 09 estudos que evidenciaram que o rastreamento realizado por profissionais da $\mathrm{AB}$, constitui como uma estratégia eficaz para prevenção e diagnóstico precoce do câncer bucal. Os estudos apontam para a necessidade de aperfeiçoamento e qualificação desses profissionais, no que concerne ao conhecimento teórico para o rastreamento do câncer bucal.

O diagnóstico situacional foi realizado com o intuito de identificar o interesse e o conhecimento desse público sobre identificação, prevenção e fatores de riscos em câncer e lesões bucais pré-malignas. Participaram desta etapa 19 cirurgiõesdentistas, atuantes na ESF de um município situado na Microrregião do Cariri, Mesorregião Sul do estado do Ceará, Nordeste, Brasil, no período de março a abril no ano de 2019. Esse quantitativo corresponde ao total de CD que compunha as equipes de ESF escolhido para o desenvolvimento do estudo.

Utilizou-se de um questionário semiestruturado, desenvolvido e validado por Cimardi (2009). A análise estatística realizada foi descritiva, com distribuição absoluta e de frequência, por meio do programa Statistical Package for lhe Social Sciences (SPSS), versão 21.0. Os dados obtidos mediante os resultados da revisão integrativa e do questionário respondidos pelos CD foram organizadas em temas para subsidiar a construção da cartilha, intitulada " Diagnóstico de lesões orais prémalignas".

Esses dados foram encaminhados a um profissional da área de designer, com experiência em elaboração de materiais educativos. As imagens foram selecionadas da internet, para servi como esboço, e após, convertidas em desenhos e trabalhadas nos programas Adobe ilustrator CS3 e para a diagramação, o Adobe InDesign CS6, originando a primeira versão da cartilha submetida à validação.

Para o processo de validação de conteúdo foram selecionados juízes que se enquadrassem a pontuação mínima de seis pontos no sistema de classificação de Fehring (1994), a saber: ter Pós-doutorado; ser doutor, com linhas de pesquisa em patologia oral, estomatologia; ser mestre; possuir capacitação (especialização/residência), nas áreas patologia oral, estomatologia; possuir artigos publicados na área; ter desenvolvidos projetos de pesquisas com abordagem em uma das seguintes áreas: patologia oral, estomatologia; ter experiência profissional em docência, pesquisa, construção e validação de tecnologias educativas, saúde coletiva.

Já os especialistas técnicos foram selecionados também conforme os critérios (Fehring, 1994): possuir Pós-doutorado na área design/marketing; ser doutor na área design/marketing; ser mestre na área design/marketing; possuir capacitação (especialização/residência) na área design/marketing; ter artigo publicado na área design/marketing; desenvolver ou ter desenvolvido projetos de pesquisa na área design/marketing; possuir experiência profissional em docência, pesquisa, construção e validação de tecnologias educativas, saúde coletiva.

Para selecionar os juízes elegíveis foram utilizadas duas estratégias: 1) busca na Plataforma Lattes utilizando o filtro de atuação profissional (Grande área: Ciências da saúde/ Área: Odontologia/ Subárea: patologia oral; estomatologia); 2) 
Mediante amostragem de rede ou bola de neve, foram convidados 25 juízes que atendiam o perfil preestabelecido, porém apenas 7 juízes aceitaram participar da pesquisa respondendo ao questionário em tempo hábil. No que se refere ao número de juízes, a literatura é diversificada e não existe um número padronizado, entretanto, Pasquali (1997) ressalta que o número de 6 (seis) a 20 (vinte) é o recomendável para o processo de validação. Outros autores, Lima et al. (2017), Albuquerque (2015), também já adotaram o número de especialistas sugerido por Pasquali (1997) para a validação de materiais educativos.

Os especialistas técnicos foram escolhidos por amostragem não probabilística intencional do tipo bola de neve. Foram convidados cinco designers gráficos para participar do processo de validação, no entanto, apenas dois responderam em tempo hábil. Assim, aos que atenderam aos critérios de elegibilidade foi enviado via e-mail um convite, no início mês de agosto de 2019. Aqueles que aceitaram participar receberam via e-mail um kit contendo: o link da Cartilha eletrônica, o Termo de Consentimento Livre e Esclarecido (TCLE), os instrumentais de avaliação, e o quadro para preencher o seu perfil.

Os especialistas da área da saúde avaliaram a cartilha de acordo com: clareza da linguagem, pertinência prática e relevância teórica. Esses critérios foram avaliados segundo grau de concordância, de forma que 1 representa "pouquíssima", 2 representa "pouca", 3 representa "média", 4 representa "muita" e 5 representa "muitíssima". Para cada página foi destinado em espaço para que os juízes pudessem escrever opiniões e sugestões.

Os especialistas técnicos avaliaram o material utilizando o instrumento Suitability Assessmente of Materials (SAM) (Doak, 1996). Este instrumento pôde propiciar que a cartilha fosse avaliada em cinco quesitos: 1) organização, 2) linguagem, 3) ilustrações gráficas, 4) motivação, 5) adequação cultural. Para cada item foi atribuída à classificação concordo totalmente, concordo parcialmente, nem concordo, nem discordo, discordo parcialmente e discordo totalmente. Para todos os especialistas foi consentido um prazo de 10 dias para a análise do material, preenchimento e envio do questionário.

Para validação de conteúdo, calculou-se o Índice de Validade de Conteúdo (IVC). O IVC das escalas de likert foi calculado com base em três equações matemáticas: S-CVI/Ave (média dos índices de validação de conteúdo para todos os índices da escala); S-CVI/UA (proporção de itens de uma escala que atinge os escores 4 "Muito" e 5 "Muitíssimo" e o I-CVI (Validade de conteúdo dos índices individuais), considerando o IVC maior ou igual a 0,78 (Polit, 2011).

Os escores do SAM foram avaliados como "Superior", valendo 2 pontos; "Adequado", 1 ponto; e "Inadequado", 0 ponto, conforme critérios objetivos incluídos no instrumento que possibilitam tanto o cálculo da média dos valores quanto a análise percentual. Assim, de acordo com a quantidade de fatores que fizeram parte do instrumento, os resultados e o percentual dos escores alcançados foram analisados, conforme orienta Doak, Doak e Root (1996), de modo que, quando a cartilha alcançar de 70 a 100\% dos escores o material educativo será considerado "Superior"; de 40 a $69 \%$, "Adequado"; e de 0 a $39 \%$, "Inadequado".

Todos os aspectos éticos que regem pesquisas envolvendo seres humanos foram respeitados e o projeto foi aprovado pelo Comitê de Ética em Pesquisa da Universidade Regional do Cariri, com o número de parecer n 3.139.515.

\section{Resultados}

Participaram da validação 09 juízes: 07 especialistas da saúde que avaliaram a cartilha em relação ao conteúdo e dois técnicos da área de comunicação/designer responsáveis pela avaliação da aparência.

A maioria dos especialistas da saúde era do sexo feminino (n=04), 06 tinham mestrado, um possuía pós-doutorado e três doutorado. Os 07 juízes possuíam publicação de artigos na temática de interesse. Ressalta que 06 dos especialistas possuíam experiência profissional em docência, pesquisa, construção e validação de tecnologias educativas, saúde coletiva.

Com relação aos critérios de classificação de Fehring (1994), todos apresentaram valores acima de 6 (máximo 15 pontos), demonstrando a adequabilidade da amostra selecionada para o estudo. Desse modo, considera-se que todos os juízes 
obtinham conhecimentos nas áreas de interesse bastante expressivo. A tabela 1 sumariza as informações sobre os juízes especialistas.

Tabela 1 - Caracterização do perfil dos juízes especialistas. Crato, Ceará, 2019.

\begin{tabular}{lcc}
\hline \multicolumn{1}{c}{ Variáveis } & F & \%* \\
\hline Critérios de Fehring & 1 & 3 \\
Possuir Pós-doutorado em uma das linhas de pesquisa: patologia oral, estomatologia & 6 & 4,2 \\
Possuir doutorado em uma das linhas de pesquisa: patologia oral, estomatologia & 5 & 85,7 \\
Possuir mestrado em uma das áreas: patologia oral, estomatologia & 7 & 71,4 \\
Possuir capacitação (especialização/residência) em uma das áreas: patologia oral, estomatologia & 6 & 85,7 \\
Possuir artigo publicado abordando um dos seguintes temas: patologia oral, estomatologia & 6 \\
Desenvolver ou ter desenvolvido projetos de pesquisa com abordagem em uma das áreas: & \multirow{2}{*}{85,7} \\
patologia oral, estomatologia & \\
Possuir experiência profissional em docência, pesquisa, construção e validação de tecnologias & \\
educativas, saúde coletiva &
\end{tabular}

Nota. $\mathrm{f}=$ frequência; $\%$ = a frequência da informação no grupo de 7 juízes. Fonte: Autores.

Os itens respondidos referentes à cartilha educativa foram inseridos, tabulados e analisados com auxílio do programa estatístico IBM SPSS. Foi realizada a análise descritiva dos dados, sendo solicitado o índice de validade de conteúdo (IVC) para cada dimensão e para cada item (I-CVI). A tabela 2 apresenta os resultados dos valores de IVC e I-CVI referentes à clareza de linguagem, pertinência prática e relevância teórica, das respectivas páginas julgadas pelos juízes.

O estudo considerou validados o item e o instrumento como um todo, que apresentaram IVC maior ou igual a 0,78 de acordo com Lynn (1986), o mesmo utilizado por outros pesquisadores, como Lima (2017).

De acordo com a tabela 2, observa-se que todas as páginas da cartilha obtiveram IVC maior do que 0,78 no aspecto clareza da linguagem, pertinência prática e relevância teórica.

Tabela 2 - Validade de Conteúdo dos Índices Individuais (I-CVI). Crato, Ceará, 2019.

\begin{tabular}{ccccc}
\hline \multirow{2}{*}{ Itens da cartilha } & \multicolumn{3}{c}{ Dimensões } & \multirow{2}{*}{ I-CVI } \\
\cline { 2 - 4 } & Clareza & Pertinência & Relevância & \\
\hline Capa & 1,0 & 1,0 & 1,0 & 1,0 \\
Folha de rosto & 1,0 & 1,0 & 1,0 & 1,0 \\
Apresentação & 1,0 & 0,9 & 1,0 & 1,0 \\
Sumário & 1,0 & 1,0 & 1,0 & 1,0 \\
Página 1 & 0,9 & 0,9 & 1,0 & 0,9 \\
Página 2 & 1,0 & 1,0 & 1,0 & 1,0 \\
Página 3 & 0,9 & 0,9 & 1,0 & 0,9 \\
Página 4 & 1,0 & 1,0 & 1,0 & 1,0 \\
Página 5 & 1,0 & 1,0 & 1,0 & 1,0 \\
Página 6 & 1,0 & 1,0 & 1,0 & 1,0 \\
Página 7 & 1,0 & 1,0 & 1,0 & 1,0 \\
Página 8 & 1,0 & 1,0 & 1,0 & 1,0 \\
Página 9 & 1,0 & 1,0 & 1,0 & 1,0 \\
Página 10 & 0,9 & 0,9 & 1,0 & 0,9 \\
Página 11 & 1,0 & 1,0 & 1,0 & 1,0 \\
Página 12 & 1,0 & 1,0 & 1,0 & 1,0 \\
Página 13 & 1,0 & 1,0 & 1,0 & 1,0 \\
Página 14 & 1,0 & 1,0 & 1,0 & 1,0 \\
Página 15 & 1,0 & 1,0 & 1,0 & 1,0 \\
Página 16 & 1,0 & 1,0 & 1,0 & 1,0 \\
Página 17 & 1,0 & 1,0 & 0,9 & 1,0 \\
Referências & 0,9 & 0,9 & 0,7 & 0,8 \\
\hline
\end{tabular}

Fonte: Autores. 
O índice de validade de conteúdo para toda a escala, levando em consideração a média de IVC para cada dimensão avaliada (clareza, pertinência e relevância), foi de 0,97 . Tanto para a clareza da linguagem quanto para a pertinência prática, os valores de IVC foram de 0,97 cada. Para a relevância teórica, o IVC foi de 0, 98. Autores apontam que o índice de validade de conteúdo é considerado adequado quando seu valor é maior ou igual a 0,8 (Alexandre, 2011). De modo geral, os resultados apontam que os valores de IVC para cada item apresentam-se de modo satisfatório, isto é, todos os itens da cartilha receberam aprovação geral dos juízes.

Em relação à validação de aparência, designers, especialistas na área de criação gráfica de conteúdo e informação, avaliaram o material da cartilha a partir dos seguintes critérios: organização do material, linguagem, ilustrações gráficas, motivação e adequação cultural. Ambos os designers apresentaram pontuação satisfatórias na classificação de Fehring (1994), o primeiro apresentando seis pontos e segundo oito pontos. Tratam-se de docentes do ensino superior de ensino, com tempo médio de formação de 4,5 anos.

Para análise de adequabilidade é apresentada na Tabela 3, os itens foram agrupados de modo que os níveis concordo totalmente/parcialmente corresponderam à avaliação "Superior"; nem concordo, nem discordo representou "Adequado"; e discordo parcialmente/totalmente constituiu "Inadequado".

Tabela 3 - Grau de concordância dos designers na avaliação da cartilha. Crato, Ceará, 2019

\begin{tabular}{|c|c|c|c|c|c|c|}
\hline & CT & $\mathbf{C P}$ & $\begin{array}{l}\text { NC/ } \\
\text { ND }\end{array}$ & DP & DT & $\%$ \\
\hline \multicolumn{7}{|l|}{ Organização } \\
\hline A capa chamou sua atenção? & 0 & 2 & 0 & 0 & 0 & \multirow{3}{*}{96} \\
\hline A sequência de conteúdo está adequada? & 2 & 0 & 0 & 0 & 0 & \\
\hline A estrutura da cartilha educativa está organizada? & 2 & 0 & 0 & 0 & 0 & \\
\hline \multicolumn{7}{|l|}{ Linguagem } \\
\hline O nível de leitura é adequado para a compreensão do público alvo (Dentistas) & 2 & 0 & 0 & 0 & 0 & \multirow{3}{*}{100} \\
\hline A maneira como as informações estão dispostas facilitam o entendimento do texto & 2 & 0 & 0 & 0 & 0 & \\
\hline O vocabulário utiliza palavras compreensíveis & 2 & 0 & 0 & 0 & 0 & \\
\hline \multicolumn{7}{|l|}{ Ilustrações Gráficas } \\
\hline A capa atrai a atenção e retrata o propósito do material & 0 & 2 & 0 & 0 & 0 & \multirow{2}{*}{96} \\
\hline A capa atrai a atenção e retrata o propósito do material & 2 & 0 & 0 & 0 & 0 & \\
\hline \multicolumn{7}{|l|}{ Motivação } \\
\hline $\begin{array}{l}\text { Ocorre interação do texto e/ou das figuras com o leitor, levando-os a refletir sobre o } \\
\text { tema, mudar de comportamento e desenvolver habilidades }\end{array}$ & 2 & 0 & 0 & 0 & 0 & \multirow{3}{*}{100} \\
\hline Os padrões de comportamento desejados são modelados ou bem demonstrados & 2 & 0 & 0 & 0 & 0 & \\
\hline $\begin{array}{l}\text { Existe a motivação à mudança de comportamento, ou seja, preocupar-se com o } \\
\text { diagnóstico precoce das lesões }\end{array}$ & 2 & 0 & 0 & 0 & 0 & \\
\hline \multicolumn{7}{|l|}{ Adequação Cultural } \\
\hline O material é culturalmente adequado à lógica, linguagem e experiência do público-alvo & 2 & 0 & 0 & 0 & 0 & \multirow{2}{*}{100} \\
\hline Apresenta imagens e exemplos adequados culturalmente & 2 & 0 & 0 & 0 & 0 & \\
\hline \multicolumn{6}{|c|}{ Escore total (SAM) } & 98,4 \\
\hline
\end{tabular}

Nota: $\mathrm{CT}=$ Concordo totalmente $\mathrm{CP}=$ Concordo parcialmente; $\mathrm{NC} / \mathrm{ND}=$ Nem concordo, nem discordo; $\mathrm{DP}=\mathrm{Discordo}$ parcialmente; $\mathrm{DT}=$ Discordo totalmente; \% = Grau de concordância. Fonte: Autores.

Como demonstrado na Tabela 3, os resultados acerca do nível de concordância dos designers, ao avaliar os critérios elencados, evidenciaram satisfação dos avaliadores com escore total de 98,4\%.

Os critérios Linguagem, Motivação e Adequação Cultural obtiveram a Concordância Total (CT) em 100\% da avaliação. Em relação aos critérios de Organização e Ilustrações Gráficas, estes foram avaliados com Concordância Parcial 
(CP) em 96 \%. Ambos os designers ao avaliarem o critério de Organização relacionado ao item "A capa chamou sua atenção", e o critério Ilustrações Gráficas do item "A capa atrai sua atenção e retrata o propósito do material”, concordaram parcialmente com esses itens. A Tabela 4 apresenta os resultados da análise de adequabilidade.

Tabela 4 - Análise de adequabilidade. Crato, Ceará, 2019.

\begin{tabular}{|c|c|c|c|c|}
\hline & 节 & 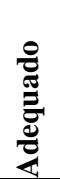 & 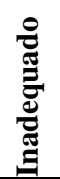 & 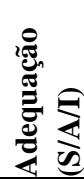 \\
\hline \multicolumn{5}{|l|}{ Organização } \\
\hline A capa chamou sua atenção? & 2 & 0 & 0 & S \\
\hline A sequência de conteúdo está adequada? & 2 & 0 & 0 & S \\
\hline A estrutura da cartilha educativa está organizada? & 2 & 0 & 0 & S \\
\hline \multicolumn{5}{|l|}{$\begin{array}{ll}\text { Linguagem } & \\
& \text { Ling }\end{array}$} \\
\hline O nível de leitura é adequado para a compreensão do público alvo (Dentistas) & 2 & 0 & 0 & S \\
\hline A maneira como as informações estão dispostas facilitam o entendimento do texto & 2 & 0 & 0 & S \\
\hline O vocabulário utiliza palavras compreensíveis & 2 & 0 & 0 & S \\
\hline \multicolumn{5}{|l|}{ Ilustrações Gráficas } \\
\hline A capa atrai a atenção e retrata o propósito do material & 2 & 0 & 0 & S \\
\hline A capa atrai a atenção e retrata o propósito do material & 2 & 0 & 0 & S \\
\hline \multicolumn{5}{|l|}{ Motivação } \\
\hline $\begin{array}{l}\text { Ocorre interação do texto e/ou das figuras com o leitor, levando-os a refletir sobre o } \\
\text { tema, mudar de comportamento e desenvolver habilidades }\end{array}$ & 2 & 0 & 0 & S \\
\hline Os padrões de comportamento desejados são modelados ou bem demonstrados & 2 & 0 & 0 & S \\
\hline $\begin{array}{l}\text { Existe a motivação à mudança de comportamento, ou seja, preocupar-se com o } \\
\text { diagnóstico precoce das lesões }\end{array}$ & 2 & 0 & 0 & S \\
\hline \multicolumn{5}{|l|}{ Adequação Cultural } \\
\hline O material é culturalmente adequado à lógica, linguagem e experiência do público-alvo & 2 & 0 & 0 & S \\
\hline Apresenta imagens e exemplos adequados culturalmente & 2 & 0 & 0 & S \\
\hline
\end{tabular}

Nota: $($ S/A/I $)=$ Superior/Adequado/Inadequado, conforme a classificação dos itens SAM. Fonte: Autores

De modo geral, os resultados apontam que os avaliadores concordam entre si acerca da adequabilidade material da cartilha. Como demonstrado em ambas às tabelas, nenhum dos dois juízes avaliou negativamente os itens. Considerando o grau de concordância, o escore calculado para o instrumento ( $\mathrm{SAM}=98,4 \%$ ) e a análise de adequabilidade, a cartilha foi considerada como "Superior" (100\%).

\section{Discussão}

A avaliação com os juízes especialistas no estudo evidenciou que a cartilha constitui uma tecnologia em saúde que reúne um conjunto de informações que pode proporcionar à construção de novos conhecimentos e outros estudos que possibilitem o seu aperfeiçoamento, pois acredita-se que nenhum conhecimento é estático (Castro, 2014).

$\mathrm{Na}$ avaliação da primeira versão da cartilha o IVC global foi de 0,97 entre os juízes especialistas. Os resultados são semelhantes ao estudo de construção e validação de um álbum seriado para o público adolescente sobre sífilis adquirida a ser utilizada como uma estratégia educativa para promoção da saúde (Santos, 2020).

A cartilha recebeu ao todo 57 sugestões, assim, após uma nova análise nos referencias teóricos utilizados para elaboração da tecnologia e mediante nova consulta nas bases de dados da literatura cientifica, foram acatadas 42 sugestões.

As sugestões, em sua maioria, foram atendidas: o título da cartilha a priore era 'Diagnóstico de lesões orais prémalignas" e após sugestões, passou a ser "'Diagnóstico das desordens orais potencialmente malignas"; alteração da palavra 
lesões por desordens; inversão de ordem das palavras diagnóstico e prognóstico; revisão ortográfica e concordância em todo o texto da cartilha; acrescentou mais um parágrafo sobre carcinoma de células escamosas oral subtipo histopatológico; exclusão de uma coluna do gráfico representando o mal prognóstico; inclusão de um fluxograma do serviço público para melhor visualização do caminho percorrido pelo paciente quando o mesmo for diagnóstico com alguma desordem oral maligna.

Uniformização da nomenclatura carcinoma de células escamosas ao longo da cartilha; inclusão de legendas nas figuras, bem como citar as alterações histológicas na leucoplasia, embora seja apenas hiperqueratose, podendo ter alguma displasia. Algumas imagens foram excluídas, de forma que valorizasse o texto. Retirou o nome "aquisição", acrescentando uma imagem referente à LPO erosiva, ao destacar a biópsia como padrão-ouro para diagnóstico.

Assim sendo, a partir da análise dos juízes especialistas, a cartilha "Diagnóstico de desordens orais potencialmente malignas" apresenta conteúdo e aparência pertinentes e válidos evidenciados pelos índices adequados de IVC referente à clareza da linguagem, pertinência prática e relevância teórica. A cartilha apresentou um nível de concordância de 98,4\%. Em pesquisas sobre validação de tecnologias os resultados os semelhantes (Moura, 2017; Sabino, 2018).

Desta forma, a cartilha instrutiva eletrônica em questão representa um material classificado como válido e confiável, com uma linguagem clara, layout e designer atraente, a qual pode ser utilizada na educação em saúde por diferentes profissionais de saúde e acadêmicos, e em variados contextos, favorecendo o seu diagnóstico precoce, elevando a sobrevida e qualidade de vida dos pacientes.

A validação com juízes com formação na área de interesse permite a construção e aplicação de instrumentos de validação, que possibilitam abordar interrogativas a respeito da relevância do conteúdo apresentado, da acessibilidade da linguagem, organização e formatação do conteúdo didático (Saraiva, 2018).

Estima-se que a cartilha é uma tecnologia educacional que reúne um conjunto de informações aplicado à construção de novos conhecimentos. Nesta pesquisa, foi desenvolvida para suscitar o conhecimento dos CD e instigá-los para o cuidado na prevenção primária e diagnóstico precoce em lesões bucais pré-malignas, a fim de evitar o surgimento do câncer bucal. Como detectado nesse estudo, estes profissionais demonstraram baixo nível de confiança para a realização de diagnósticos $(78,9 \%)$.

Percebe-se que as tecnologias em educação favorecem a disseminação do conhecimento com finalidade de expandir a autonomia do indivíduo em prol da melhoria da qualidade de vida, e para que seja atingida essa finalidade, é necessário avaliar $\mathrm{o}$ artefato em todos aspectos de sua linguagem para se ter a segurança que o material aplicado ao público seja compreendido em toda sua dimensão, facilitando a tomada de decisões (Albuquerque, 2016).

O uso de cartilhas educacionais, pautadas na educação em saúde devem ser entendidas como um importante instrumento na prevenção de doenças, bem como na promoção de saúde, desde que sejam desenvolvidas estratégias que possibilitem a melhoria nas condições de vida da população (Ramos, 2018).

O cuidado a ser prestado indica o tipo de tecnologia a ser aplicada na prática, nesse contexto, as cartilhas educativas podem ser classificadas como cuidados baseados em tecnologias leve-duras, pois apropriam-se de conhecimentos e saberes estruturados para sua formulação, subsidiando um canal para a troca de informações, objetivando a internalização dos saberes e práticas (Honorato, 2015).

Acredita-se que esses resultados satisfatórios em relação à concordância tenham sido possibilitados pela cautela e estudo metodológico aplicado ao se elaborar o material educativo. O trabalho foi realizado em conjunto, de modo multiprofissional, considerando os fundamentos científicos evidenciados na literatura e conhecimentos dos especialistas, respeitando os critérios essenciais para elaboração de um material educativo, desde a elaboração do conteúdo, escolha de cores em tons claros para que a leitura não ficasse cansativa e ainda obtivesse uma estética favorável, e modo de comunicação em 
que se disponibilizou o texto, utilizando-os da forma mais clara e objetiva possível, linguagem acessível ao público-alvo, disposição de informação em tópicos, esquemas, gráficos e ilustrações que pudessem informar e motivar os leitores.

Aponta-se como limitação a não adesão dos juízes especialistas no processo de avaliação do material elaborado. No entanto, apesar do número reduzido de juízes foi possível realizar a fase de validação com êxito, seguindo os critérios mínimos encontrados na literatura para o desenho desde tipo de estudo.

\section{Conclusão}

A cartilha elaborada foi validada por especialistas que a consideram uma tecnologia importante e eficaz para profissionais quanto ao diagnóstico e identificação de fatores de riscos para prevenção de lesões bucais malignas. O material tem como principal contribuição o esclarecimento de dúvidas e a reunião de um conjunto de conhecimentos para cirurgiões dentistas, de modo, a instiga-los para o desenvolvimento de práticas preventivas para evitar o surgimento do câncer bucal. Espera-se que a tecnologia possa ser incorporada na Atenção Básica como forma de promover a orientação e capacitação dos profissionais cirurgiões dentistas.

\section{Agradecimentos}

À Coordenação de Aperfeiçoamento de Pessoal de Nível Superior (CAPES) pela concessão de bolsa ao Programa de Pós-Graduação em Enfermagem, da Universidade Regional do Cariri.

\section{Referências}

Alexandre, N. M. C \& Coluci, M. Z. O. (2011). Validade de conteúdo nos processos de construção e adaptação de instrumentos de medidas. Ciência e Saúde Coletiva. 16, 3061-3068.

Albuquerque, A. F. L. L. et al. (2016). Tecnologia para o autocuidado da saúde sexual e reprodutiva de mulheres estomizadas. Revista Brasileira de Enfermagem. 69 (6), 1164-1171.

Brasil. (2018). Ministério da Saúde. A saúde bucal no Sistema Único de saúde. Ministério da Saúde.

Castro, A. N. P \& Lima Junior, E. M. (2013). Desenvolvimento e validação de cartilha para pacientes vítimas de queimaduras. Sociedade Brasileira de Queimaduras. 13(2), 103-13.

Carvalho, D. P., Rodrigues, R. M \& Braz, E. (2013). Estratégias de educação em saúde direcionadas a cuidadores durante a internação. Revista Acta Paul de Enfermagem. 26(5), 455-459.

Cimardi, A. C. B. S. (2009). Câncer de boca: conhecimento e prática de cirurgiões-dentistas do Estado de Santa Catarina. 2009. 112f. Dissertação (Pós Graduação em Odontologia) - Universidade Federal de Santa Catarina, Santa Catarina.

Doak, C. C., Doak, L. G \& Root, J. H. (1996). Teaching pacients wint low literacy skills. (2a ed.), J. B. Lippincott, 1996.

Echer, I. C. (2005). Elaboração de manuais de orientação para o cuidado em saúde. Revista Latino-Americana de Enfermagem. 13 (5), $754-757$.

Fehring, R. J. (1994). The Fehring model. In: Carool - Jonhnson RM, Paquete M. Classification of nursing diagnoses: tenth conference. Philadelphia: J. B. Linppincott.

Falcão, M. M. L. et al. (2010). Conhecimento dos cirurgiões-dentistas em relação ao câncer bucal. Revista Gaúcha de Odontologia, Porto Alegre. 58 (1), 2733.

Honorato, D. Z. et al. (2015). O uso de tecnologias em saúde na consulta: uma análise reflexiva. Revista Interdisciplinar. 8 (1), $234-239$.

Inca. (2014). Instituto Nacional de Câncer. Estimativa 2016: Incidência de Câncer no Brasil. INCA.

Inca. (2020). Instituto Nacional de Câncer. Boca. http://www2.inca.gov.br/wps/wcm/connect/tiposdecancer/site/home/boca/definicao.

Lynn, M. R. (2017). Determanation and quantification of content validity. Nursing Research. 35 (9), 382-385.

Lima, A. C. M. A. C. et al. (2017). Development and validation of a booklet for prevention of vertical HIV transmission. Acta Paulista de Enfermagem. 30 (2), 181-189.

Moura, I. H., Silva, A. F. R., Rocha, A. E. S. H., Lima, L. H. O., Moreira, T. M. M \& Silva ARV. (2017). Construction and validation of educational materials for the prevention of metabolic syndrome in adolescents. Rev Latino-Am Enfermagem. 25:e2934. 10.1590/1518-8345.2024.2934 
Research, Society and Development, v. 10, n. 14, e189101421684, 2021

(CC BY 4.0) | ISSN 2525-3409 | DOI: http://dx.doi.org/10.33448/rsd-v10i14.21684

Oms. (2005). Organizaçao Mundial da Saúde. Pathology and Genetics of Head and Neck Tumours: classification of tumours. Lyon: IARC Press.

Polit, D. F., Beck, C. T. (2011). Fundamentos de pesquisa em enfermagem: avaliação de evidências para as práticas da enfermagem. 7. ed. Porto Alegre: Artmed.

Ramos, R. T. et al. (2017). Leucoplasia Oral: conceitos e repercussões clínicas. Revista Brasileira de Odontologia, 74 (1), 51-5.

Santos, S. B., Ramos, J. L. S., Machado, A. P. A., Lopes, M. T. N., Abreu, L. C \& Bezerra, I. M. P. (2020). Tecnologia educativa para adolescentes: construção e validação de álbum seriado sobre sífilis adquirida. Rev Bras Promoç Saúde. 33:9970. 10.5020/18061230.2020.9970

Sabino, L. M., Ferreira, A. M., Joventino, E. S., Lima, F. E., Penha, J. C., Lima, K. F. et al. (2018). Elaboration and validation of a reader on childhood diarrhea prevention. Acta Paul Enferm. 31(3):233-9. 10.1590/1982-0194201800034

Saraiva, N. C. G., Medeiros, C. C. M \& Araújo, T. L. (2018). Validação de álbum seriado para a promoção do controle de peso corporal infantil. Revista Latino-Americana de Enfermagem. 26 (1), 2998.

Silva, B. S. et al. (2018). Conhecimento dos Cirurgiões-Dentistas da rede pública sobre câncer bucal. Revisão de Literatura. 12 (42), $1018-1026$.

Silveira, E. J. D 7 et al. (2014). Avaliação clínica e histopatológica e hábitos associados ao surgimento de leucoplasias e eritroplasias orais. Jornal Brasileiro de Patologia e Medicina Laboratorial. 50(2), 144-9. 